\title{
Physical Models for Particle Tracking Simulations in the RF Gap
}

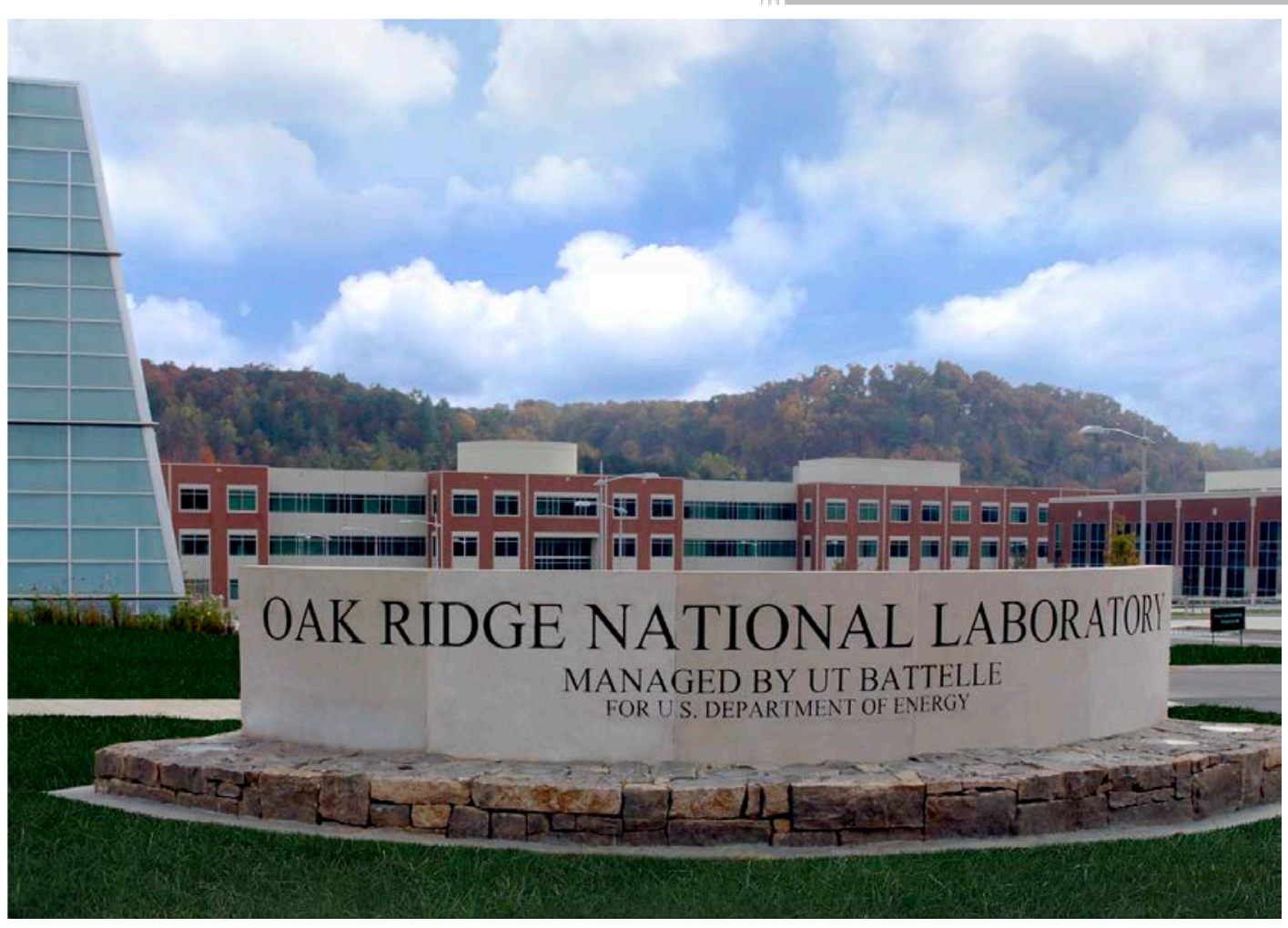

Approved for public release. Distribution is unlimited.
Andrei Shishlo

Jeff Holmes

June 2015 


\section{DOCUMENT AVAILABILITY}

Reports produced after January 1, 1996, are generally available free via US Department of Energy (DOE) SciTech Connect.

Website http://www.osti.gov/scitech/

Reports produced before January 1, 1996, may be purchased by members of the public from the following source:

National Technical Information Service

5285 Port Royal Road

Springfield, VA 22161

Telephone 703-605-6000 (1-800-553-6847)

TDD 703-487-4639

Fax 703-605-6900

E-mail info@ntis.gov

Website http://www.ntis.gov/help/ordermethods.aspx

Reports are available to DOE employees, DOE contractors, Energy Technology Data Exchange representatives, and International Nuclear Information System representatives from the following source:

Office of Scientific and Technical Information

PO Box 62

Oak Ridge, TN 37831

Telephone 865-576-8401

Fax 865-576-5728

E-mail reports@osti.gov

Website http://www.osti.gov/contact.html

- $\quad$ This report was prepared as an account of work sponsored by an agency of the United States Government. Neither the United States Government nor any agency thereof, nor any of their employees, makes any warranty, express or implied, or assumes any legal liability or responsibility for the accuracy, completeness, or usefulness of any information, apparatus, product, or process disclosed, or represents that its use would not infringe privately owned rights. Reference herein to any specific commercial product, process, or service by trade name, trademark, manufacturer, or otherwise, does not necessarily constitute or imply its endorsement, recommendation, or favoring by the United States Government or any agency thereof. The views and opinions of authors expressed herein do not necessarily state or reflect those of the United States Government or any agency thereof. 


\title{
OAK RIDGE NATIONAL LABORATORY
}

MANAGED BY UT-BATTELLE FOR THE DEPARTMENT OF ENERGY

\section{Technical Note}

Research Accelerator Division

Accelerator Physics Team
From: Andrei Shishlo, Jeff Holmes

Number: ORNL/TM-2015/247

Date: June 4, 2015

Subject: Physical Models for Particle Tracking Simulations in the RF Gap

\begin{abstract}
This document describes the algorithms that are used in the PyORBIT code to track the particles accelerated in the Radio-Frequency cavities. It gives the mathematical description of the algorithms and the assumptions made in each case. The derived formulas have been implemented in the PyORBIT code. The necessary data for each algorithm are described in detail.
\end{abstract}

\section{Contents}

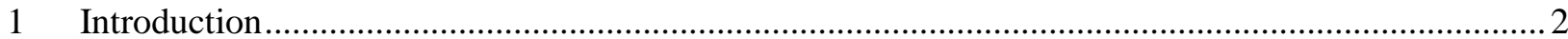

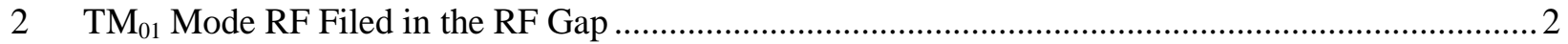

3 Particle Coordinate Transformations in the RF Gap........................................................................... 5

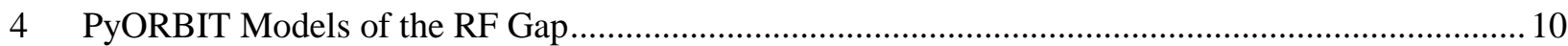

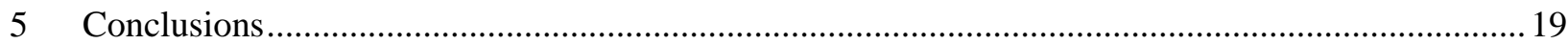

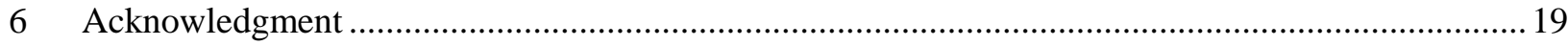

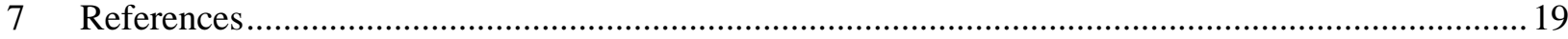

\section{Introduction}

Each linac simulation code has a model (or several models) for tracking charged particles through the electro-magnetic field of a radio-frequency (RF) cavity. Sometime these models are very simple, such as a set of thin RF gaps in the Trace3D code, or they can be very complicated, such as tracking through the time-dependent 3-dimensional (3D) fields of the cavity with a full account of self-induced spacecharge forces in the Track code. The details of the algorithms and their implementations are frequently hidden from users, especially for the closed source codes. This makes it difficult to benchmark these codes against one another and to analyze what simplifying assumptions could be used in different 
situations to speed up the simulation process without reducing the quality of the results. This paper describes in detail the simulation models that are used in the PyORBIT code to calculate particle tracking through the RF cavities.

We will start our description with the electro-magnetic field in the RF cavity and the forces acting upon the particle accelerating in this field. In general we will follow the approach of the textbook by Thomas P. Wrangler, "RF Linear Accelerators" (WILEY, 2008 2nd edition) [1]. However, we will make somewhat different simplifying assumptions than those made in the textbook, so that our formulas will differ.

\section{$2 \mathrm{TM}_{01}$ Mode RF Filed in the RF Gap}

In general, an RF cavity is an array of accelerating gaps (there may be only one) arranged at certain distances to provide a synchronous acceleration from one gap to another. The electric field in the gap is defined by Maxwell's equations in vacuum

$$
\nabla^{2} \vec{E}-\frac{1}{c^{2}} \frac{\partial^{2} \vec{E}}{\partial t^{2}}=0 \quad \nabla^{2} \vec{B}-\frac{1}{c^{2}} \frac{\partial^{2} \vec{B}}{\partial t^{2}}=0
$$

In the case of axial symmetry, the longitudinal electric field for the $\mathrm{TM}_{01}$ mode in the RF cavity satisfies the following equation in the cylindrical coordinate system

$$
\frac{\partial^{2} E_{z}(r, z, t)}{\partial z^{2}}+\frac{1}{r} \frac{\partial E_{z}(r, z, t)}{\partial r}+\frac{\partial^{2} E_{z}(r, z, t)}{\partial r^{2}}-\frac{1}{c^{2}} \frac{\partial^{2} E_{z}(r, z, t)}{\partial t^{2}}=0
$$

The fixed-frequency solution of Eq. (2.2) is

$$
E_{z}(r, z, t)=E_{z}(r, z) \cdot \cos (\omega \cdot t+\varphi)
$$

where

$$
E_{z}(r, z) \rightarrow 0 \text { for } z \rightarrow \pm \infty
$$

Let's represent $E_{z}(r, z)$ as a sum of symmetric and asymmetric parts with respect to $z=0$

$$
E_{z}(r, z)=E_{z}^{(S)}(r, z)+E_{z}^{(A)}(r, z)
$$

where these two parts are defined as

$$
\begin{aligned}
& E_{z}^{(S)}(r, z)=\frac{1}{2}\left(E_{z}(r, z)+E_{z}(r,-z)\right) \\
& E_{z}^{(A)}(r, z)=\frac{1}{2}\left(E_{z}(r, z)-E_{z}(r,-z)\right)
\end{aligned}
$$


To write a solution of Eq. (2.2) in a desirable form, let's introduce a Fourier transformation for the symmetric and asymmetric parts

$$
\begin{aligned}
& V_{0} \cdot T(r, k)=\int_{-\infty}^{+\infty} E_{z}^{(S)}(r, z) \cdot \cos (k \cdot z) d z \\
& V_{0} \cdot S(r, k)=\int_{-\infty}^{+\infty} E_{z}^{(A)}(r, z) \cdot \sin (k \cdot z) d z
\end{aligned}
$$

where

$$
V_{0}=\int_{-\infty}^{+\infty} E_{z}(r=0, z) d z
$$

The inverse transformations are

$$
\begin{aligned}
& E_{z}^{(S)}(r, z)=\frac{V_{0}}{2 \cdot \pi} \int_{-\infty}^{+\infty} T(r, z) \cdot \cos (k \cdot z) d k \\
& E_{z}^{(A)}(r, z)=\frac{V_{0}}{2 \cdot \pi} \int_{-\infty}^{+\infty} S(r, z) \cdot \sin (k \cdot z) d k
\end{aligned}
$$

Combining Eqs. (2.9), (2.5), (2.3) and (2.2), and taking into account that "sin" and "cos" are linearly independent functions, we obtain equations for $T(r, k)$ and $S(r, k)$

$$
\begin{aligned}
& \frac{\partial^{2} T(r, k)}{\partial r^{2}}+\frac{1}{r} \cdot \frac{\partial T(r, k)}{\partial r}+K^{2} \cdot T(r, k)=0 \\
& \frac{\partial^{2} S(r, k)}{\partial r^{2}}+\frac{1}{r} \cdot \frac{\partial S(r, k)}{\partial r}+K^{2} \cdot S(r, k)=0
\end{aligned}
$$

where

$$
K^{2}=\frac{\omega^{2}}{c^{2}}-k^{2}
$$

Equation (2.10) is Bessel's Equation, and it has different solutions depending on the sign of $K^{2}$

$$
\begin{aligned}
& T(r, k)=T(k) \cdot\left\{\begin{array}{l}
J_{0}(|K| \cdot r) \\
I_{0}(|K| \cdot r)
\end{array}\right\} \text { for } \begin{array}{r}
K^{2}=\frac{\omega^{2}}{c^{2}}-k^{2} \geq 0 \\
K^{2}=\frac{\omega^{2}}{c^{2}}-k^{2} \leq 0
\end{array} \\
& S(r, k)=S(k) \cdot\left\{\begin{array}{l}
J_{0}(|K| \cdot r) \\
I_{0}(|K| \cdot r)
\end{array}\right\} \text { for } \begin{array}{r}
K^{2}=\frac{\omega^{2}}{c^{2}}-k^{2} \geq 0 \\
K^{2}=\frac{\omega^{2}}{c^{2}}-k^{2} \leq 0
\end{array}
\end{aligned}
$$


The solutions, Eq. (2.12), must be finite for $r \rightarrow 0$, so that no Neumann or Hankel functions appear here.

Finally, the formula for the longitudinal electric field is

$$
E_{z}(r, z, t)=\frac{V_{0}}{2 \cdot \pi} \int_{-\infty}^{+\infty} d k(T(k) \cdot \cos (k \cdot z)+S(k) \cdot \sin (k \cdot z)) \cdot\left\{\begin{array}{l}
J_{0}(|K| \cdot r) \\
I_{0}(|K| \cdot r)
\end{array}\right\} \cdot \cos (\omega \cdot t+\varphi)
$$

where

$$
\begin{aligned}
& T(k)=\frac{1}{V_{0}} \int_{-\infty}^{+\infty} E_{z}(r=0, z) \cdot \cos (k \cdot z) \cdot d z \\
& S(k)=\frac{1}{V_{0}} \int_{-\infty}^{+\infty} E_{z}(r=0, z) \cdot \sin (k \cdot z) \cdot d z
\end{aligned}
$$

The formula in Eq. (2.13) also gives us the z-axis derivatives of the longitudinal field

$$
\begin{gathered}
\frac{\partial E_{z}(r, z)}{\partial z}=\frac{V_{0}}{2 \cdot \pi} \int_{-\infty}^{+\infty} d k\left\{k \cdot(-T(k) \cdot \sin (k \cdot z)+S(k) \cdot \cos (k \cdot z)) \cdot\left\{\begin{array}{c}
J_{0}(|K| \cdot r) \\
I_{0}(|K| \cdot r)
\end{array}\right\}\right\} \\
\frac{\partial^{2} E_{z}(r, z)}{\partial z^{2}}=\frac{V_{0}}{2 \cdot \pi} \int_{-\infty}^{+\infty} d k\left\{\left(-k^{2}\right) \cdot(T(k) \cdot \cos (k \cdot z)+S(k) \cdot \sin (k \cdot z)) \cdot\left\{\begin{array}{c}
J_{0}(|K| \cdot r) \\
I_{0}(|K| \cdot r)
\end{array}\right\}\right\}
\end{gathered}
$$

In the $\mathrm{TM}_{01}$ mode, Gauss' law may be used to express the radial component of the electric field in terms of the longitudinal component as

$$
E_{r}(r, z, t)=-\frac{1}{r} \int_{0}^{r} \frac{\partial E_{z}\left(r^{\prime}, z, t\right)}{\partial z} \cdot r^{\prime} \cdot d r^{\prime}
$$

Therefore the analog of the Eq. (2.13) for $E_{r}(r, z, t)$ is

$$
E_{r}(r, z, t)=\frac{V_{0}}{2 \cdot \pi} \int_{-\infty}^{+\infty} d k\left\{\frac{k}{|K|}(T(k) \cdot \sin (k \cdot z)-S(k) \cdot \cos (k \cdot z)) \cdot\left\{\begin{array}{c}
J_{1}(|K| \cdot r) \\
I_{1}(|K| \cdot r)
\end{array}\right\}\right\} \cdot \cos (\omega \cdot t+\varphi)
$$

To derive Eq. (2.18) we used the following formula for Bessel functions

$$
\frac{1}{r} \int_{0}^{r} r^{\prime} \cdot\left\{\begin{array}{c}
J_{0}\left(|K| \cdot r^{\prime}\right) \\
I_{0}\left(|K| \cdot r^{\prime}\right)
\end{array}\right\} \cdot d r^{\prime}=\frac{1}{|K|} \cdot\left\{\begin{array}{c}
J_{1}(|K| \cdot r) \\
I_{1}(|K| \cdot r)
\end{array}\right\}
$$

In the $\mathrm{TM}_{01}$ mode, the magnetic field has only one non-zero component, $B_{\theta}(r, z, t)$, which can be found from Ampere's Law 


$$
\oint_{L} \vec{B} d \vec{l}=\mu_{0} \cdot \varepsilon_{0} \cdot \oint_{S} \frac{\partial \vec{E}}{\partial t} d \vec{s} \quad \text { where } \quad c=\frac{1}{\sqrt{\mu_{0} \cdot \varepsilon_{0}}}
$$

This gives us the following formula

$$
B_{\theta}(r, z, t)=-\frac{\omega}{c^{2} \cdot r}\left[\int_{0}^{r} E_{z}\left(r^{\prime}, z\right) \cdot r^{\prime} \cdot d r^{\prime}\right] \cdot \sin (\omega \cdot t+\varphi)
$$

And finally (after using Eq. (2.19))

$$
B_{\theta}(r, z, t)=-\frac{V_{0} \cdot \omega}{2 \cdot \pi \cdot c^{2}} \int_{-\infty}^{+\infty} d k\left\{\frac{1}{|K|}(T(k) \cos (k \cdot z)+S(k) \sin (k \cdot z)) \cdot\left\{\begin{array}{l}
J_{1}(|K| r) \\
I_{1}(|K| r)
\end{array}\right\}\right\} \sin (\omega \cdot t+\varphi)(2.22)
$$

Equations (2.13), (2.18), and (2.22) define the RF gap field in a convenient form. We will show how to use them to calculate the particle coordinates transformations in the tracking code.

\section{Particle Coordinate Transformations in the RF Gap}

Here, we use Equations 13,18 , and 22 for $E_{z}(r, z, t), E_{r}(r, z, t)$, and $B_{\theta}(r, z, t)$ to calculate momentum kicks for particles moving through these fields. In general we will follow the recipes from Thomas P. Wrangler's book "RF Linear Accelerators" (WILEY, $20082^{\text {nd }}$ edition, p. 39) [1]. The formulas in this Tech Note are generalizations of those presented in Reference [1] to incorporate possible asymmetry of the field in the RF gap

$$
S(k) \neq 0
$$

In Wrangler's textbook the geometric center of the gap is chosen in such way that the asymmetric transit time factor $S(k)$ is equal to zero. In our case this assumption is impractical, because in our simulation codes the position of the gap center is determined by the $k$ value that corresponds to the energy of the synchronous particle. Therefore, we have to constantly modify our accelerator lattice during tracking simulations, which significantly complicates the structure of the code. In our implementation of Wrangler's approach we will not assume that this asymmetric transit time factor is zero. There is also another difference related to correction terms for $r^{\prime}$ which will be described later.

In performing the coordinate transformations for the particles moving through the RF field, we will assume the straight-line approximation for the particle trajectories. This approximation makes sense for most cases, because the particles in the simulated bunches move primarily along the z-axis. The angles between the $z$-axis and the particle velocities are usually less than several milliradians. The exact analysis of the applicability of this approximation should be done separately for each real case. The ultimate check would be a benchmark with the exact tracking of the particles through the real 3D field of the RF cavity. 
The equation of the straight-line trajectory of the particle, moving parallel to the $z$-axis, is

$$
\begin{aligned}
& r=\text { const } \\
& z=c \cdot \beta \cdot t
\end{aligned}
$$

\subsection{Energy Gain and Phase Advance}

To calculate the energy gain $\Delta W$ of the particle moving along the z-axis we integrate $E_{z}(r, z, t)$ along the trajectory of Eq. (3.2)

$$
\Delta W=q \cdot \int_{-\infty}^{+\infty} E_{z}\left(r, z, t=\frac{Z}{c \cdot \beta}\right) \cdot d z
$$

It is worth commenting here that the infinite limits on the integration are completely general because the function $E_{z}(r, z, t)$ strongly approaches zero outside the confines of the RF gap. After we substitute Eq. (2.13) into Eq. (3.3) and take into account

$$
\cos (\omega \cdot t+\varphi)=\cos (\omega \cdot t) \cdot \cos (\varphi)-\sin (\omega \cdot t) \cdot \sin (\varphi)
$$

we obtain

$$
\Delta W=\frac{q V_{0}}{2 \cdot \pi} \cdot\left\{\begin{array}{l}
+\int_{-\infty}^{+\infty} d k \cdot T(k) \cdot\left(\int_{-\infty}^{+\infty} \cos (k z) \cdot \cos \left(\frac{\omega}{c \cdot \beta} z\right) d z\right) \cdot \cos (\phi) \\
-\int_{-\infty}^{+\infty} d k \cdot T(k) \cdot\left(\int_{-\infty}^{+\infty} \cos (k z) \cdot \sin \left(\frac{\omega}{c \cdot \beta} z\right) d z\right) \cdot \sin (\phi) \\
+\int_{-\infty}^{+\infty} d k \cdot S(k) \cdot\left(\int_{-\infty}^{+\infty} \sin (k z) \cdot \cos \left(\frac{\omega}{c \cdot \beta} z\right) d z\right) \cdot \cos (\phi) \\
-\int_{-\infty}^{+\infty} d k \cdot S(k) \cdot\left(\int_{-\infty}^{+\infty} \sin (k z) \cdot \sin \left(\frac{\omega}{c \cdot \beta} z\right) d z\right) \cdot \sin (\phi)
\end{array}\right\} \cdot\left\{\begin{array}{l}
J_{0}(K \mid r) \\
I_{0}(K \mid r)
\end{array}\right\}
$$

The integrals

$$
\int_{-\infty}^{+\infty} \cos (k z) \cdot \sin \left(\frac{\omega}{c \cdot \beta} z\right) d z=0 \text { and } \int_{-\infty}^{+\infty} \sin (k z) \cdot \cos \left(\frac{\omega}{c \cdot \beta} z\right) d z=0
$$

are 0 , because they are integrals of odd functions. The integrals of the even functions in Eq. (3.5) evaluate to Dirac delta functions: 


$$
\begin{aligned}
& \int_{-\infty}^{+\infty} \cos (k z) \cos \left(\frac{\omega}{c \beta} z\right) d z=\frac{1}{2} \int_{-\infty}^{+\infty}\left(\cos \left(k z-\frac{\omega}{c \beta} z\right)+\cos \left(k z+\frac{\omega}{c \beta} z\right)\right) d z=\pi\left(\delta\left(k-\frac{\omega}{c \beta}\right)+\delta\left(k+\frac{\omega}{c \beta}\right)\right) \\
& \int_{-\infty}^{+\infty} \sin (k z) \sin \left(\frac{\omega}{c \beta} z\right) d z=\frac{1}{2} \int_{-\infty}^{+\infty}\left(\cos \left(k z-\frac{\omega}{c \beta} z\right)-\cos \left(k z+\frac{\omega}{c \beta} z\right)\right) d z=\pi\left(\delta\left(k-\frac{\omega}{c \beta}\right)-\delta\left(k+\frac{\omega}{c \beta}\right)\right)
\end{aligned}
$$

Using the properties of the $T(k)$ and $S(k)$ transit time factor functions (Eq. (2.14))

$$
T(-k)=T(k) \text { and } S(-k)=-S(k)
$$

and integrating over $k$ in Eq. (3.5) we get

$$
\Delta W(k, \varphi)=q V_{0} I_{0}\left(\frac{\omega}{c \gamma \beta} r\right) \cdot(T(k) \cdot \cos (\varphi)-S(k) \cdot \sin (\varphi)), \text { where } k=\frac{\omega}{c \beta}=\frac{2 \pi \cdot f_{R F}}{c \beta}
$$

and it is appropriate to use $I_{0}(K)$, the modified Bessel function, when the particle velocity $\beta<1$. The Bessel function argument also can be represented in the following form

$$
\frac{\omega}{c \gamma \beta} r=\sqrt{k^{2}-k_{0}^{2}} \cdot r \text { where } k=\frac{\omega}{c \beta} \text { and } k_{0}=\frac{\omega}{c}
$$

We could assume the particle's phase (or equivalently time of arrival or distance to the synchronous particle) to be constant, but this violates Liouville's theorem and emittance conservation. This problem arises because of the energy dependence of $T(k)$ and $S(k)$ in Eq. (3.9). One way to avoid this difficulty, as suggested in 1963 at a Linac Conference, would be to ignore this energy dependence and use the transit time factors in Eq. (3.9) at the synchronous energy. Another solution [Carne et al.] is to introduce a phase change at the RF gap crossing [2]. To calculate this phase change $\Delta \phi$ we can use the following

$$
\begin{aligned}
& \Delta W=-\frac{\partial H}{\partial \varphi} \\
& \Delta \varphi=+\frac{\partial H}{\partial W} \text { where } H \text { is the particle Hamiltonian }
\end{aligned}
$$

The Hamiltonian in Eq. (3.11) is defined as

$$
H(k, \varphi)=-q V_{0} I_{0}\left(\sqrt{k^{2}-k_{0}^{2}} \cdot r\right) \cdot(T(k) \cdot \sin (\varphi)+S(k) \cdot \cos (\varphi))
$$

By using Eq. (3.12) and the first relation in Eq. (3.11) we get the correct energy gain in Eq. (3.9). To obtain $\Delta \varphi$ we use the second part of Eq. (3.11) and the relationship between $W$ and $\boldsymbol{k}$, which can be obtained from $W=\gamma m c^{2}$ and Eq. (3.9) for $\boldsymbol{k}$ in terms of $\boldsymbol{\beta}$. 


$$
\Delta \varphi=+\frac{\partial H}{\partial W}=\frac{\partial H}{\partial k} \cdot \frac{\partial k}{\partial W}=-\frac{k}{m c^{2} \gamma^{3} \beta^{2}} \cdot \frac{\partial H}{\partial k}
$$

Using

$$
I_{0}^{\prime}(x)=I_{1}(x) \text { and } \frac{d}{d k}\left(\sqrt{k^{2}-k_{0}^{2}}\right)=\frac{k}{\sqrt{k^{2}-k_{0}^{2}}}=\gamma
$$

we get

$$
\Delta \varphi=\frac{q V_{0} \cdot k}{m c^{2} \gamma^{3} \beta^{2}}\left\{\begin{array}{l}
I_{0}\left(\sqrt{k^{2}-k_{0}^{2}} \cdot r\right) \cdot\left(T^{\prime}(k) \cdot \sin (\varphi)+S^{\prime}(k) \cdot \cos (\varphi)\right)+ \\
+\gamma \cdot r \cdot I_{1}\left(\sqrt{k^{2}-k_{0}^{2}} \cdot r\right) \cdot(T(k) \cdot \sin (\varphi)+S(k) \cdot \cos (\varphi))
\end{array}\right\}
$$

The formulas (3.9) and (3.15) give us the transformations for the longitudinal coordinates of the particles passing through the RF gap, and these transformations satisfy Liouville's theorem. During the calculations we assumed that all parameters related to the energy of the particle (like $\beta, \gamma$, and $k$ ) are defined at the initial energy. If desired, one could use the values at the middle of the gap, or even use an iteration process to calculate these parameters. We will not do this here because, we will use a longitudinal slicing of the field and applying the formulas (3.9) and (3.15) separately at each longitudinal slice.

Finally, we note that the values defined by Eq. (3.15) are usually small, especially for the highest energies, so the approximation with constant transit time factors could give us satisfactory results for the case of a small energy spread in the bunch.

\subsection{Transverse Coordinate Transformations}

The change in the transverse momentum of the particle moving through the RF gap field will come from the transverse component of the electric field $E_{r}(r, z, t)$ and the azimuthal component of the magnetic field $B_{\theta}(r, z, t)$

$$
\Delta p_{r}=q \cdot \int_{-\infty}^{+\infty}\left(E_{r}\left(r, z, t=\frac{z}{c \cdot \beta}\right)-c \beta \cdot B_{\theta}\left(r, z, t=\frac{z}{c \cdot \beta}\right)\right) \frac{d z}{c \beta}
$$

By using the formulas (2.12), (2.18), (3.6), (3.7), (3.8) and the following identities

$$
\begin{aligned}
& \cos \left(\omega \cdot \frac{Z}{c \beta}+\varphi\right)=\cos \left(\omega \cdot \frac{Z}{c \beta}\right) \cdot \cos (\varphi)-\sin \left(\omega \cdot \frac{Z}{c \beta}\right) \cdot \sin (\varphi) \\
& \sin \left(\omega \cdot \frac{Z}{c \beta}+\varphi\right)=\sin \left(\omega \cdot \frac{Z}{c \beta}\right) \cdot \cos (\varphi)+\cos \left(\omega \cdot \frac{Z}{c \beta}\right) \cdot \sin (\varphi)
\end{aligned}
$$


we get

$$
\begin{gathered}
\Delta p_{r}=\Delta p_{r}^{\left(E_{r}\right)}+\Delta p_{r}^{\left(B_{\theta}\right)} \\
\Delta p_{r}^{\left(E_{r}\right)}=-q V_{0} \cdot \frac{\gamma}{c \beta} \cdot I_{1}\left(\sqrt{k^{2}-k_{0}^{2}} \cdot r\right) \cdot(T(k) \cdot \sin (\varphi)+S(k) \cdot \cos (\varphi)) \\
\Delta p_{r}^{\left(B_{\theta}\right)}=q V_{0} \cdot \frac{\gamma \beta}{c} \cdot I_{1}\left(\sqrt{k^{2}-k_{0}^{2}} \cdot r\right) \cdot(T(k) \cdot \sin (\varphi)+S(k) \cdot \cos (\varphi))
\end{gathered}
$$

From the formulas (3.18) and (3.19) we can see that defocusing from the electric field dominates focusing from the magnetic field at low energies (low $\beta$ ), and it will more and more compensated at the higher energies.

The final expression for the transverse kick is

$$
\Delta p_{r}=-\frac{q V_{0}}{c \beta \gamma} \cdot I_{1}\left(\sqrt{k^{2}-k_{0}^{2}} \cdot r\right) \cdot(T(k) \cdot \sin (\varphi)+S(k) \cdot \cos (\varphi))
$$

Equation (3.20) satisfies Liouville's theorem (remember that $r=$ const ) because the right hand side is independent of $\Delta p_{r}$ in the first order.

For small values of $\sqrt{k^{2}-k_{0}^{2}} \cdot r<<1$

$$
I_{1}\left(\sqrt{k^{2}-k_{0}^{2}} \cdot r\right) \approx \frac{1}{2} \sqrt{k^{2}-k_{0}^{2}} \cdot r \text { where } \sqrt{k^{2}-k_{0}^{2}}=\frac{2 \pi}{\gamma \beta \lambda} ; \lambda=\frac{2 \pi c}{\omega}
$$

and Eq. (3.20) is transformed to

$$
\Delta p_{r}=-\frac{\pi q V_{0}}{c \lambda \beta^{2} \gamma^{2}} \cdot r \cdot(T(k) \cdot \sin (\varphi)+S(k) \cdot \cos (\varphi)) \text { for } \sqrt{k^{2}-k_{0}^{2}} \cdot r<<1
$$

Equations (3.20) and (3.22) are the same Eqs. (7.39) and 7.29), respectively, in Sects. 7.4 and 7.5 of Ref. [1 under the assumption of a symmetric RF gap field $S(k)=0$.

We cannot apply the transformation (3.20) directly to the coordinates of the particles in the PyORBIT bunch, because we operate with $x, x^{\prime}$ and $y, y^{\prime}$ coordinates, not $r, r^{\prime}$. According to Eq. (3.20) the momentum kicks are directed along $\vec{n}_{r}=\frac{x \cdot \vec{e}_{x}+y \cdot \vec{e}_{y}}{r}$ where $\vec{e}_{x}$ and $\vec{e}_{y}$ are the $\mathrm{x}$ and $\mathrm{y}$ unit vectors

$$
\Delta \vec{p}=\Delta p_{r} \cdot \vec{n}_{r} \text { and } \Delta p_{x}=\frac{x}{r} \Delta p_{r} ; \Delta p_{y}=\frac{y}{r} \Delta p_{r}
$$

For $\mathrm{x}^{\prime}$ and $\mathrm{y}^{\prime}$ the transformation formulas are 


$$
\begin{gathered}
\Delta p_{x}=\Delta\left(\gamma \beta m c \cdot x^{\prime}\right)=m c \cdot(\gamma \beta)_{\text {out }} \cdot x_{\text {out }}^{\prime}-m c \cdot(\gamma \beta)_{\text {in }} \cdot x_{\text {in }}^{\prime} \\
\Delta p_{y}=\Delta\left(\gamma \beta m c \cdot y^{\prime}\right)=m c \cdot(\gamma \beta)_{\text {out }} \cdot y_{\text {out }}^{\prime}-m c \cdot(\gamma \beta)_{\text {in }} \cdot y_{\text {in }}^{\prime} \\
x_{\text {out }}^{\prime}=\frac{(\gamma \beta)_{\text {in }}}{(\gamma \beta)_{\text {out }}} \cdot x_{\text {in }}^{\prime}-\frac{1}{(\gamma \beta)_{\text {out }}} \cdot \frac{x}{r} \cdot \frac{q V_{0}}{m c^{2} \beta \gamma} \cdot I_{1}\left(\sqrt{k^{2}-k_{0}^{2}} \cdot r\right) \cdot(T(k) \cdot \sin (\varphi)+S(k) \cdot \cos (\varphi)) \\
y_{\text {out }}^{\prime}=\frac{(\gamma \beta)_{\text {in }}}{(\gamma \beta)_{\text {out }}} \cdot y_{\text {in }}^{\prime}-\frac{1}{(\gamma \beta)_{\text {out }}} \cdot \frac{y}{r} \cdot \frac{q V_{0}}{m c^{2} \beta \gamma} \cdot I_{1}\left(\sqrt{k^{2}-k_{0}^{2}} \cdot r\right) \cdot(T(k) \cdot \sin (\varphi)+S(k) \cdot \cos (\varphi))
\end{gathered}
$$

The transformations (3.25) give the transverse coordinate changes for particles passing through the RF gap. These equations complete our physics model. We are not going consider correction terms to account for components of motion non-parallel to the z-axis. Although this is done in many books, these corrections are based on an assumption of axial symmetry (they deal with $r$ and $r^{\prime}$ only) of particle motion inside the bunch

$$
\frac{x^{\prime}}{y^{\prime}}= \pm \frac{x}{y}
$$

This is not true for realistic bunches.

In the next section we will discuss the concrete implementation of the coordinate transformation formulas, Eqs. (3.9), (3.15), and (3.25).

\section{PyORBIT Models of the RF Gap}

There are many potential ways to implement the formulas given by Eqs. (3.9), (3.15), and (3.25). In this section we will describe several RF gap models that were implemented in the PyORBIT code. Let's consider features common for all or almost all implementations.

First, the integral limits in the z-variable integration in all formulas were set equal to infinity, which is unrealistic. From here on we will assume that the RF gap has a limited length from $z_{-1}$ to $Z_{+1}$ with the center at $z_{0}$. We choose the center to be the position of the maximum value of the electric field on the axis: $\left|E_{z}\left(r=0, z_{0}\right)\right|=\max$. The motion of the particle before and after the center is treated as a free motion, and the change in the coordinates is applied at the center. During the motion of the particle through the drifts before and after the center of the gap, space charge kicks may be applied to the bunch. This model is called a "thin length" RF gap model.

Second, all models are z-type (instead of t-type) with respect to the longitudinal coordinate treatment in the bunch. This is more convenient for implementation, but less realistic than using time as the independent coordinate. This simplification could create differences between our results and more realistic simulations when collective interactions cause significant changes in the bunch during the propagation through the gap. It is very difficult to estimate the effect of these approximations in 
advance, but for routine situations at the design parameters of existing linacs we should be very close to reality.

Third, PYORBIT uses a non-canonical pair of longitudinal coordinates $d E$ and $z$ (energy deviation and the longitudinal position relative to that of the synchronous particle) instead of the canonical pair $d E$ and $\varphi=\omega \cdot t$. This means that after the RF gap transformation there is an immediate change in the bunch longitudinal size, but the longitudinal emittance is preserved. It is the same as the situation for $\left(x, x^{\prime}\right)$ and $\left(y, y^{\prime}\right)$ pairs instead of $\left(x, p_{x}\right)$ and $\left(y, p_{y}\right)$, which give us formulas (3.25).

\subsection{Simplified Matrix Model}

Very often we want to benchmark a Particle-In-Cell code (like PyORBIT) with an envelope code such as Trace3D, the Online Model in OpenXAL, or the envelope-tracking model in the TraceWin code, etc. To do so we have to simplify our formulas for the particle coordinate transformations in order to extract the linear part. After that, the PIC code can replace the envelope code, and by comparing the results with more realistic simulations we can estimate the effects of non-linearity in the RF cavities. In PyORBIT, this linear model is called the Simplified Matrix Model.

There are several assumptions in the Simplified Matrix Model in the PyORBIT code. First, we assume that RF gap field distribution is symmetric along the $z$-axis relative to $z=0$. This means that

$$
S(k)=0
$$

in all formulas. Then, we replace $V_{0} \cdot T\left(k=k_{s}\right)$ with $E_{0} T L$, which is the maximum energy gain achievable in the RF gap. The value $k_{s}=1 / c \beta_{s}$ is the wave number of the synchronous particle

$$
V_{0} \cdot T\left(k=k_{s}\right)=E_{0} T L
$$

The trigonometric functions are expanded linearly about the synchronous particle

$$
\begin{aligned}
& \cos (\varphi)=\cos \left(\varphi_{s}+\varphi-\varphi_{s}\right) \approx \cos \left(\varphi_{s}\right)-\sin \left(\varphi_{s}\right) \cdot\left(\varphi-\varphi_{s}\right) \\
& \sin (\varphi)=\sin \left(\varphi_{s}+\varphi-\varphi_{s}\right) \approx \sin \left(\varphi_{s}\right)+\cos \left(\varphi_{s}\right) \cdot\left(\varphi-\varphi_{s}\right)
\end{aligned}
$$

where $\varphi_{s}$ is the phase of the synchronous particle for the RF gap. With these assumptions we obtain the following modification of the formulas (3.9), (3.15), and (3.25)

$$
\begin{array}{r}
\varphi-\varphi_{s}=-\frac{\omega}{c \beta_{s}} \cdot z \\
\lambda=\frac{2 \pi c}{\omega}
\end{array}
$$




$$
\begin{aligned}
& \Delta W_{s}=W_{s}^{(\text {out })}-W_{s}^{(i n)}=q \cdot E_{0} T L \cdot \cos \left(\varphi_{s}\right) \\
& \Delta W-\Delta W_{s}=W^{(\text {out })}-W_{s}^{(\text {out })}-\left(W^{(\text {in })}-W_{s}^{(\text {in })}\right)=d E_{\text {out }}-d E_{\text {in }}=-q \cdot E_{0} T L \cdot \sin \left(\varphi_{s}\right) \cdot\left(\varphi-\varphi_{s}\right) \\
& d E_{\text {out }}-d E_{\text {in }}=q \cdot \frac{\omega}{c \cdot \beta_{s}^{(i n)}} \cdot E_{0} T L \cdot \sin \left(\varphi_{s}\right) \cdot Z_{\text {in }} \\
& \varphi_{s}^{(\text {out })}=\varphi_{s}^{(\text {in })} \text { and } \varphi^{(\text {out })}-\varphi_{s}^{(\text {out })}=\varphi^{(\text {in })}-\varphi_{s}^{(\text {in })} \\
& Z^{(\text {out })}=\frac{\beta_{s}^{(\text {out })}}{\beta_{s}^{(i n)}} Z^{(i n)} \\
& x_{\text {out }}^{\prime}=\frac{(\gamma \beta)_{s}^{(i n)}}{(\gamma \beta)_{s}^{(o u t)}} \cdot x_{\text {in }}^{\prime}-x \cdot q \cdot \frac{\pi}{m c^{2} \cdot \lambda \cdot\left(\beta^{2} \gamma^{2}\right)_{s}^{(i n)} \cdot(\gamma \beta)_{s}^{(o u t)}} \cdot E_{0} T L \cdot \sin \left(\varphi_{s}\right) \\
& y_{\text {out }}^{\prime}=\frac{(\gamma \beta)_{s}^{(\text {in })}}{(\gamma \beta)_{s}^{(\text {out })}} \cdot y_{\text {in }}^{\prime}-y \cdot q \cdot \frac{\pi}{m c^{2} \cdot \lambda \cdot\left(\beta^{2} \gamma^{2}\right)_{s}^{(i n)} \cdot(\gamma \beta)_{s}^{(\text {out })}} \cdot E_{0} T L \cdot \sin \left(\varphi_{s}\right)
\end{aligned}
$$

The formulas (4.1.11) do not have contributions from $x \cdot\left(\varphi-\varphi_{s}\right)$ or $y \cdot\left(\varphi-\varphi_{s}\right)$, because we consider only linear terms.

The negative sign in Eq. (4.1.4) is determined by the choice of bunch coordinates in PyORBIT. Positive longitudinal coordinates correspond to the head of the bunch. We will use the same convention in all our models.

The Simplified Matrix Model has only two parameters to define the RF gap, namely, the maximum energy gain and the phase of the synchronous particle. This makes it very convenient for fast and simple estimation of the beam parameters in the linac.

\subsection{Base RF Gap Model}

The base RF gap model keeps the simplicity of the matrix model, but it adds the non-linear coordinate transformations with respect to the phase of the particle and its distance from the axis. In the base model we still assume that the field in the gap satisfies

$$
S(k)=0
$$

and the parameter $E_{0} T L$ defines all RF gap dynamics

$$
V_{0} \cdot T\left(k=k_{s}\right)=E_{0} T L
$$


But here we do not use the linear expansions for $\cos (\varphi), \sin (\varphi), I_{1}\left(\sqrt{k^{2}-k_{0}^{2}} \cdot r\right)$, and $I_{0}\left(\sqrt{k^{2}-k_{0}^{2}} \cdot r\right)$

$$
\begin{gathered}
W_{\text {out }}-W_{\text {in }}=q \cdot E_{0} T L \cdot I_{0}\left(\frac{\omega}{c(\gamma \beta)_{\text {in }}} r\right) \cdot \cos \left(\varphi_{\text {in }}\right) \\
\varphi_{\text {out }}=\varphi_{\text {in }} \\
z^{(\text {out })}=\frac{\beta_{\text {sut })}^{(\text {on })}}{\beta_{s}^{(\text {in })}} \\
x_{\text {out }}^{\prime}=\frac{(\gamma \beta)_{\text {in }}}{(\gamma \beta)_{\text {out }}} \cdot x_{\text {in }}^{\prime}-\frac{1}{(\gamma \beta)_{\text {out }}} \cdot \frac{x}{r} \cdot \frac{q \cdot E_{0} T L}{m c^{2}(\beta \gamma)_{\text {in }}} \cdot I_{1}\left(\frac{\omega}{c \cdot(\gamma \beta)_{\text {in }}} \cdot r\right) \cdot \sin \left(\varphi_{\text {in }}\right) \\
y_{\text {out }}^{\prime}=\frac{(\gamma \beta)_{\text {in }}}{(\gamma \beta)_{\text {out }}} \cdot y_{\text {in }}^{\prime}-\frac{1}{(\gamma \beta)_{\text {out }}} \cdot \frac{y}{r} \cdot \frac{q \cdot E_{0} T L}{m c^{2}(\beta \gamma)_{\text {in }}} \cdot I_{1}\left(\frac{\omega}{c \cdot(\gamma \beta)_{\text {in }}} \cdot r\right) \cdot \sin \left(\varphi_{\text {in }}\right)
\end{gathered}
$$

Comparing the results from this model with those from the Matrix model allows us to estimate the contributions of non-linearities arising from this more realistic approach.

\subsection{Transit Time Factors RF Gap Model}

The Transit Time Factors Model (TTF Model) directly uses all Eqs. (3.9), (3.15), and (3.25) from Part 3 of this paper. The model assumes that the transit time factor functions $T(k), S(k), T^{\prime}(k)$, and $S^{\prime}(k)$ were calculated in advance, and that they are readily accessible for use in the calculations.

$$
\begin{gathered}
W_{\text {out }}=W_{\text {in }}+q V_{0} I_{0}\left(\frac{\omega}{c \cdot(\gamma \beta)_{\text {in }}} r\right) \cdot\left(T\left(k_{\text {in }}\right) \cdot \cos \left(\phi_{\text {in }}\right)-S\left(k_{\text {in }}\right) \cdot \sin \left(\phi_{\text {in }}\right)\right) \text { where } k=\frac{\omega}{c \beta}=\frac{2 \pi \cdot f_{R F}}{c \beta} \\
\varphi_{\text {out }}=\varphi_{\text {in }}+\frac{q V_{0} \cdot \omega}{m c^{3} \cdot(\gamma \beta)_{\text {in }}^{3}}\left\{\begin{array}{l}
I_{0}\left(\frac{\omega}{c \cdot(\gamma \beta)_{\text {in }}} \cdot r\right) \cdot\left(T^{\prime}\left(k_{\text {in }}\right) \cdot \sin \left(\varphi_{\text {in }}\right)+S^{\prime}\left(k_{\text {in }}\right) \cdot \cos \left(\varphi_{\text {in }}\right)\right)+ \\
\left.+\gamma_{\text {in }} \cdot r \cdot I_{1}\left(\frac{\omega}{c \cdot(\gamma \beta)_{\text {in }}} \cdot r\right) \cdot\left(T\left(k_{\text {in }}\right) \cdot \sin \left(\varphi_{\text {in }}\right)+S\left(k_{\text {in }}\right) \cdot \cos \left(\varphi_{\text {in }}\right)\right)\right\} \\
x_{\text {out }}^{\prime}=\frac{(\gamma \beta)_{\text {in }}}{(\gamma \beta)_{\text {out }}} x_{\text {in }}^{\prime}-\frac{1}{(\gamma \beta)_{\text {out }}} \frac{x}{r} \frac{q V_{0}}{m c^{2}(\beta \gamma)_{\text {in }}} I_{1}\left(\frac{\omega}{c \cdot(\gamma \beta)_{\text {in }}} r\right)\left(T\left(k_{\text {in }}\right) \cdot \sin \left(\varphi_{\text {in }}\right)+S\left(k_{\text {in }}\right) \cdot \cos \left(\varphi_{\text {in }}\right)\right) \\
y_{\text {out }}^{\prime}=\frac{(\gamma \beta)_{\text {in }}}{(\gamma \beta)_{\text {out }}} y_{\text {in }}^{\prime}-\frac{1}{(\gamma \beta)_{\text {out }}} \frac{y}{r} \frac{q V_{0}}{m c^{2}(\beta \gamma)_{\text {in }}} I_{1}\left(\frac{\omega}{c \cdot(\gamma \beta)_{\text {in }}} r\right)\left(T\left(k_{\text {in }}\right) \cdot \sin \left(\varphi_{\text {in }}\right)+S\left(k_{\text {in }}\right) \cdot \cos \left(\varphi_{\text {in }}\right)\right)
\end{array}\right.
\end{gathered}
$$

where the particle phase and the z-axis position are related by the Eq. (4.1.4) 


$$
\varphi-\varphi_{s}=-\frac{\omega}{c \beta_{s}} \cdot z
$$

and $\varphi_{s}$ and $c \beta_{s}$ are the phase and velocity of the synchronous particle.

Of course there can be modifications of the formulas (4.3.1-3) arising from the numerical scheme. For instance, we could replace $k_{\text {in }}$ in (4.3.3) or (4.3.2) with the $k$ defined at the energy in the middle of the RF gap $W=\left(W_{\text {out }}+W_{\text {in }}\right) / 2$. This will change the final results, but the same or similar change could be probably be achieved by changing the RF amplitude of the cavity (the parameter $V_{0}$ ). It is an interesting question, if it is possible to resolve this ambiguity by an experiment, but this question is beyond the scope of this work.

\subsection{Three Point TTF Model}

The models that were considered above all treat the RF gap as one element. If we want to be more precise we can slice up the RF gap longitudinally and consider the tracking of the bunch through each slice separately. In this case our assumption about the absence of transverse motion will be more realistic. Fig. 4.4.1 shows the $E_{z}(z)$ field divided into four longitudinal parts, each divided in half and containing a central point. Let's consider the transit time factor for a single slice.

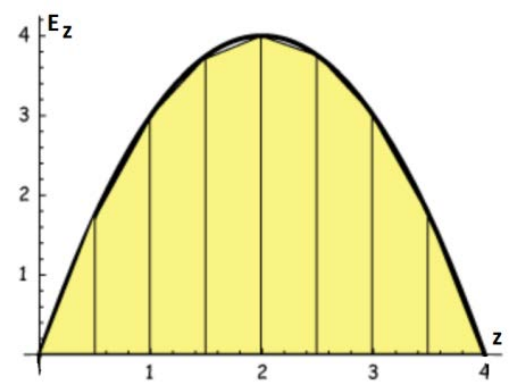

Fig. 4.4.1. The longitudinal electric field on the z-axis divided onto 4 parts.

Start with the representation of the electric field on the z-axis. Assume that we know the value of the field $E_{-1}, E_{0}$, and $E_{+1}$ for three point of the slice $-\Delta z, 0$, and $+\Delta z$. The field is shown in Fig. 4.4.2.

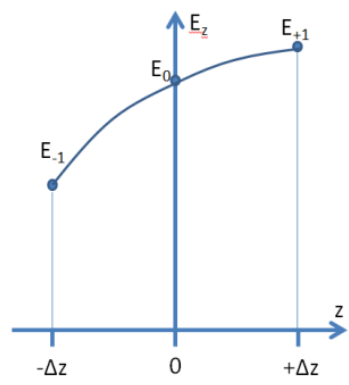


Fig. 4.4.2. The electric field in one longitudinal slice.

The field can be approximated by the second order polynomial

$$
E_{z}(z)=\widetilde{E}_{0} \cdot\left(1+a \cdot z+b \cdot z^{2}\right)
$$

where the parameters are defined by

$$
\left\{\begin{array}{c}
\tilde{E}_{0}=E_{0} \\
a=\frac{E_{+1}-E_{-1}}{2 E_{0} \Delta z} \\
b=\frac{E_{+1}+E_{-1}-2 E_{0}}{2 E_{0}(\Delta z)^{2}}
\end{array}\right.
$$

The integral of the $E_{z}(z)$ for the slice is

$$
V_{0}=\tilde{E}_{0} \cdot \int_{-\Delta z}^{+\Delta z}\left(1+a \cdot z+b \cdot z^{2}\right) d z=\widetilde{E}_{0} \cdot\left(2 \Delta z+\frac{2}{3} \cdot b \cdot \Delta z^{3}\right)
$$

The transit time factors are, according to Eq. (2.14),

$$
\begin{gathered}
T(k)=\frac{1}{V_{0}} \int_{-\Delta z}^{+\Delta z} E_{z}(z) \cdot \cos (k \cdot z) d z \\
S(k)=\frac{1}{V_{0}} \int_{-\Delta z}^{+\Delta z} E_{z}(z) \cdot \sin (k \cdot z) d z \\
T(k)=\frac{2 \sin (k \Delta z)}{k \cdot\left(2 \Lambda z+\frac{2}{3} \cdot b \cdot \Delta z^{3}\right)} \cdot\left(1+b \Delta z^{2}-\frac{2 b}{k^{2}}+\frac{2 b k \Delta z}{k^{2}} \cot (k \Delta z)\right) \\
S(k)=\frac{2 \cdot a \cdot \sin (k \Delta z)}{k^{2} \cdot\left(2 \Lambda z+\frac{2}{3} \cdot b \cdot \Delta z^{3}\right)} \cdot(1-k \Lambda z \cdot \cot (k \Delta z))
\end{gathered}
$$

The derivatives of these transit time functions are

$$
T^{\prime}(k)=-\frac{2 \sin (k \Delta z)}{k \cdot\left(2 \Delta z+\frac{2}{3} \cdot b \cdot \Delta z^{3}\right)} \cdot\left(\frac{1}{k}\left(1+3 b \Delta z^{2}-\frac{6 b}{k^{2}}\right)-\Delta z \cdot \cot (k \Delta z) \cdot\left(1+b \Delta z^{2}-\frac{6 b}{k^{2}}\right)\right)
$$




$$
S^{\prime}(k)=\frac{2 a \sin (k \Lambda z)}{k \cdot\left(2 \Lambda z+\frac{2}{3} \cdot b \cdot \Delta z^{3}\right)} \cdot\left(\Delta z^{2}-\frac{2}{k^{2}}+\Delta z \cdot \cot (k \Lambda z) \cdot \frac{2}{k}\right)
$$

Using these expressions for $T(k), S(k), T^{\prime}(k)$, and $S^{\prime}(k)$ we calculate the coordinate transformation with Eqns. (4.3.1-3) from the previous subsection. The only data that we need for this model is the function $E_{z}(z)$. The longitudinal slicing is also convenient for the space charge kick calculations.

\subsection{Direct Tracking through 3D RF Gap Field Model}

At this moment, direct particle tracking through the electro-magnetic field is the final RF gap model in PyORBIT. The 3D and time dependent field should be calculated in advance by a suitable electro-magnetic code. The 3D grids for the longitudinal and radial electric and the azimuthal magnetic field components are read from an external file or files, and they are kept in the memory. These fields are used during the solution of the equation of the particle's motion subject to the Lorentz force

$$
\frac{d \vec{p}}{d t}=q \cdot(E+c \cdot \vec{\beta} \times \vec{B})
$$

To solve the differential equation (4.5.1) this model uses the classical Runge-Kutta (RK4) method. During the tracking, the model uses the time variable instead of the s-parameter, so the coordinates of the particles in the bunch must be translated to the absolute momentum and position vectors prior to tracking, and they must then be transformed back after the RF gap tracking is finished. To apply the space charge kicks the 3D field can be sliced longitudinally, so the s-t-coordinate transformations must be done each time we track a bunch through a particular slice. This model is the slowest model for simulations, so it should be used only when really necessary.

\subsection{OpenXAL RF Gap Model}

OpenXAL is a library of Java classes for high level physics control room applications. It includes an envelope model for an accelerator, which is called the Online Model (OM). The RF gap transformation is a part of $\mathrm{OM}$, so it is important to describe the $\mathrm{OM}$ algorithm in detail. For the envelope model, in addition to tracking the synchronous particle we must keep only the linear part of the RF gap forces. We will not assume that the transit time factors are constants as we did for the Matrix and Base models. Using the formulas (3.9) and (3.15), the energy and time of arrival of the synchronous particle are

$$
W_{S}^{(\text {out })}-W_{S}^{(i n)}=q V_{0} \cdot\left(T\left(k_{S}^{(i n)}\right) \cdot \cos \left(\varphi_{S}^{(i n)}\right)-S\left(k_{S}^{(i n)}\right) \cdot \sin \left(\varphi_{S}^{(i n)}\right)\right)
$$




$$
\begin{aligned}
& \phi_{S}^{(o u t)}-\phi_{S}^{(\dot{m})}=\frac{q V_{0} \omega}{m c^{3}\left(\gamma_{S} \beta_{S}\right)_{\bar{m}}^{3}}\left(T^{\prime}\left(k_{S}^{(\dot{m})}\right) \cdot \sin \left(\phi_{S}^{(\dot{m})}\right)+S^{\prime}\left(k_{S}^{(\bar{m} n)}\right) \cdot \cos \left(\phi_{S}^{(\dot{m}))}\right)\right) \\
& \varphi_{S}=\omega \cdot t_{S}
\end{aligned}
$$

For other particles we ignore the radial dependence and use the same formulas as Eqs. (4.6.1) and (4.6.2), removing subscript " $S$ " denoting the synchronous particle. However, we do retain the linear variation of the TTFs $T(k), S(k), T^{\prime}(k), S^{\prime}(k)$ and $\cos \left(\varphi_{S}+\varphi-\varphi_{S}\right), \sin \left(\varphi_{S}+\varphi-\varphi_{S}\right)$

$$
\begin{aligned}
& T(k)=T\left(k_{S}+k-k_{S}\right) \approx T\left(k_{S}\right)+T^{\prime}\left(k_{S}\right) \cdot\left(k-k_{S}\right)=T\left(k_{S}\right)-T^{\prime}\left(k_{S}\right) \cdot \frac{\omega}{c \beta_{S}} \cdot \frac{\Delta \beta}{\beta_{S}} \\
& S(k)=S\left(k_{S}+k-k_{S}\right) \approx S\left(k_{S}\right)+S^{\prime}\left(k_{S}\right) \cdot\left(k-k_{S}\right)=S\left(k_{S}\right)-S^{\prime}\left(k_{S}\right) \cdot \frac{\omega}{c \beta_{S}} \cdot \frac{\Delta \beta}{\beta_{S}} \\
& T^{\prime}(k) \approx T^{\prime}\left(k_{S}\right)-T^{\prime \prime}\left(k_{S}\right) \cdot \frac{\omega}{c \beta_{S}} \cdot \frac{\Delta \beta}{\beta_{S}} \\
& S^{\prime}(k) \approx S^{\prime}\left(k_{S}\right)-S^{\prime \prime}\left(k_{S}\right) \cdot \frac{\omega}{c \beta_{S}} \cdot \frac{\Delta \beta}{\beta_{S}} \\
& \frac{1}{(\gamma \beta)^{3}}=\frac{1}{\left(\gamma_{S} \beta_{S}\right)^{3}}+\frac{d}{d \beta}\left(\frac{1}{(\gamma \beta)^{3}}\right) \cdot \Delta \beta=\frac{1}{\left(\gamma_{S} \beta_{S}\right)^{3}}-\frac{3}{\gamma_{S} \beta_{S}^{3}} \cdot \frac{\Delta \beta}{\beta_{S}} \\
& \cos (\varphi)=\cos \left(\varphi_{s}+\varphi-\varphi_{s}\right) \approx \cos \left(\varphi_{s}\right)-\sin \left(\varphi_{s}\right) \cdot\left(\varphi-\varphi_{s}\right) \\
& \sin (\varphi)=\sin \left(\varphi_{s}+\varphi-\varphi_{s}\right) \approx \sin \left(\varphi_{s}\right)+\cos \left(\varphi_{s}\right) \cdot\left(\varphi-\varphi_{s}\right) \\
& \Delta E=W-W_{S} \approx m c^{2} \gamma_{S}^{3} \beta_{S}^{2} \frac{\Delta \beta}{\beta_{S}} \\
& \Delta \varphi=\left(\varphi-\varphi_{S}\right)=-\omega \frac{Z}{c \beta_{S}}
\end{aligned}
$$

The last equation depends on the definition of the "Head-Tail" for the OpenXAL Online Model, which is the same as the PyORBIT's definition.

And after we linearize the equations, keeping only the first order in $\Delta \beta, \Delta E$ and $\Delta \varphi$, we get 


$$
\begin{aligned}
& \left(\frac{\Delta \beta}{\beta_{S}}\right)_{\text {out }}=\left\{\begin{array}{l}
\left(\frac{\left(\gamma_{S}^{3} \beta_{S}^{2}\right)_{\text {in }}}{\left(\gamma_{S}^{3} \beta_{S}^{2}\right)_{\text {out }}}-\frac{1}{\left(\gamma_{S}^{3} \beta_{S}^{2}\right)_{\text {out }}} \frac{q V_{0} \omega}{m c^{3} \beta_{S}^{(i n)}}\left(T^{\prime}\left(k_{S}^{(i n)}\right) \cos \left(\varphi_{S}^{(i n)}\right)-S^{\prime}\left(k_{S}^{(i n)}\right) \sin \left(\varphi_{S}^{(i n)}\right)\right)\right)\left(\frac{\Delta \beta}{\beta_{S}}\right)_{\text {in }} \\
+\frac{1}{\left(\gamma_{S}^{3} \beta_{S}^{2}\right)_{\text {out }}} \frac{q V_{0} \omega}{m c^{3} \beta_{S}^{(i n)}}\left(T\left(k_{S}^{(i n)}\right) \sin \left(\varphi_{S}^{(i n)}\right)+S\left(k_{S}^{(i n)}\right) \cos \left(\varphi_{S}^{(i n)}\right)\right) \cdot z
\end{array}\right\} \\
& \Delta \phi_{\text {out }}-\Delta \phi_{i n}=\frac{q V_{0} \omega}{m c^{3}\left(\gamma_{s} \beta_{s}\right)_{i n}^{3}}\left\{\begin{array}{l}
\left(\begin{array}{l}
3 \gamma_{S}^{2}\left(T^{\prime}\left(k_{S}^{(i n)}\right) \sin \left(\phi_{S}^{(i n)}\right)+S^{\prime}\left(k_{S}^{(i n)}\right) \cos \left(\phi_{S}^{(i n)}\right)\right)+ \\
+\frac{\omega}{c \beta_{S}^{(i n)}}\left(T^{\prime \prime}\left(k_{S}^{(i n)}\right) \sin \left(\phi_{S}^{(i n)}\right)+S^{\prime \prime}\left(k_{S}^{(i n)}\right) \cos \left(\phi_{S}^{(i n)}\right)\right)
\end{array}\right) \cdot \frac{\Delta \beta}{\beta_{S}^{(i n)}}+ \\
+\left(T^{\prime}\left(k_{S}^{(i n)}\right) \cos \left(\phi_{S}^{(i n)}\right)-S^{\prime}\left(k_{S}^{(i n)}\right) \sin \left(\phi_{S}^{(i n)}\right)\right) \cdot \Delta \phi_{i n}
\end{array}\right\} \\
& z_{\text {out }}=\frac{\beta_{S}^{(\text {out })}}{\beta_{S}^{(i n)}} z_{\text {in }}+\frac{q V_{0} \beta_{S}^{(o n t)}}{m c^{2}\left(\gamma_{s} \beta_{s}\right)_{i n}^{3}}\left\{\begin{array}{l}
\left(\begin{array}{l}
3 \gamma_{S}^{2}\left(T^{\prime}\left(k_{S}^{(i n)}\right) \sin \left(\phi_{S}^{(i n)}\right)+S^{\prime}\left(k_{S}^{(i n)}\right) \cos \left(\phi_{S}^{(i n)}\right)\right)+ \\
+\frac{\omega}{c \beta_{S}^{(i n)}}\left(T^{\prime \prime}\left(k_{S}^{(i n)}\right) \sin \left(\phi_{S}^{(i n)}\right)+S^{\prime \prime}\left(k_{S}^{(i n)}\right) \cos \left(\phi_{S}^{(i n)}\right)\right)
\end{array}\right) \cdot \frac{\Delta \beta}{\beta_{S}^{(i n)}}+ \\
+\frac{\omega}{c \beta_{S}^{(i n)}}\left(T^{\prime \prime}\left(k_{S}^{(i n)}\right) \cos \left(\phi_{S}^{(i n)}\right)-S^{\prime}\left(k_{S}^{(i n)}\right) \sin \left(\phi_{S}^{(i n)}\right)\right) \cdot z_{\text {in }}
\end{array}\right\}
\end{aligned}
$$

The formulas for the transverse components are taken from Eq. (3.25) in the limit $r->0$.

$$
\begin{aligned}
& x_{\text {out }}^{\prime}=\frac{(\gamma \beta)_{S}^{(i n)}}{(\gamma \beta)_{S}^{(\text {out })}} \cdot x_{\text {in }}^{\prime}-\frac{1}{(\gamma \beta)_{S}^{(\text {out })}} \frac{q V_{0} \omega}{2 m c^{3}\left((\beta \gamma)_{S}^{(i n)}\right)^{2}}\left(T\left(k_{S}^{(i n)}\right) \cdot \sin \left(\varphi_{S}^{(i n)}\right)+S\left(k_{S}^{(i n)}\right) \cdot \cos \left(\varphi_{S}^{(i n)}\right)\right) \cdot x \\
& y_{\text {out }}^{\prime}=\frac{(\gamma \beta)_{S}^{(i n)}}{(\gamma \beta)_{S}^{(\text {out })}} \cdot y_{\text {in }}^{\prime}-\frac{1}{(\gamma \beta)_{S}^{(\text {out })}} \frac{q V_{0} \omega}{2 m c^{3}\left((\beta \gamma)_{S}^{(i n)}\right)^{2}}\left(T\left(k_{S}^{(i n)}\right) \cdot \sin \left(\varphi_{S}^{(i n)}\right)+S\left(k_{S}^{(i n)}\right) \cdot \cos \left(\varphi_{S}^{(i n)}\right)\right) \cdot y
\end{aligned}
$$

We can simplify Eqs. (4.6.9) and (4.6.11) by assuming the constant TTF approximation and eliminating all TTF derivatives.

$$
\begin{gathered}
\left(\frac{\Delta \beta}{\beta_{S}}\right)_{\text {out }}=\left\{\left(\frac{\left(\gamma_{S}^{3} \beta_{S}^{2}\right)_{\text {in }}}{\left(\gamma_{S}^{3} \beta_{S}^{2}\right)_{\text {out }}}\right)\left(\frac{\Delta \beta}{\beta_{S}}\right)_{\text {in }}+\frac{1}{\left(\gamma_{S}^{3} \beta_{S}^{2}\right)_{\text {out }}} \frac{q V_{0} \omega}{m c^{3} \beta_{S}^{(i n)}}\left(T\left(k_{S}^{(i n)}\right) \sin \left(\varphi_{S}^{(i n)}\right)+S\left(k_{S}^{(i n)}\right) \cos \left(\varphi_{S}^{(i n)}\right)\right) \cdot z\right\} \\
Z_{\text {out }}=\frac{\beta_{S}^{(\text {out })}}{\beta_{S}^{(i n)}} Z_{\text {in }}
\end{gathered}
$$




\section{Conclusions}

We derived the formulas that are implemented in the PyORBIT code for accelerating RF gaps. At this moment, the total number of the models for the RF gap is five. We did not compare the tracking results for these models because that is beyond the scope of this paper.

\section{Acknowledgment}

The present work was prepared by Oak Ridge National Laboratory for UT-BATTELLE, LLC under contract DE-AC05-00OR22725 for the U.S. DEPARTMENT OF ENERGY.

\section{References}

1. Thomas P. Wrangler "RF Linear Accelerator" (WILEY, $20082^{\text {nd }}$ edition)

2. P. Lapostolle, Proton Linear Accelerators: A theoretical and Historical Introduction, LA-11601-MS, 1989. 